Revue d'histoire de l'Amérique française

BRS REVUE D.HISTOIRE DE L'AMÉRIQUE FRANÇAISE

\title{
Un dossier de plantation de Saint-Domingue (1745-1829)
}

\section{Roger Massio}

Volume 5, numéro 4, mars 1952

URI : https://id.erudit.org/iderudit/802135ar

DOI : https://doi.org/10.7202/802135ar

Aller au sommaire du numéro

Éditeur(s)

Institut d'histoire de l'Amérique française

ISSN

0035-2357 (imprimé)

1492-1383 (numérique)

Découvrir la revue

Citer cet article

Massio, R. (1952). Un dossier de plantation de Saint-Domingue (1745-1829).

Revue d'histoire de l'Amérique française, 5(4), 542-577.

https://doi.org/10.7202/802135ar d'utilisation que vous pouvez consulter en ligne.

https://apropos.erudit.org/fr/usagers/politique-dutilisation/ 


\title{
UN DOSSIER DE PLANTATION DE SAINT-DOMINGUE (1745-1829)
}

\author{
AVANT-PROPOS
}

Cette étude, objet d'une thèse complémentaire, soutenue devant la Faculté des Lettres de Toulouse, en juin 1950, sous le titre "Les biens et le genre de vie d'une famille noble, à Saint-Domingue et en Bigorre (1745-1836)" a suscité diverses critiques. On nous a notamment reproché et à juste titre d'ailleurs, d'avoir tenté de réaliser une synthèse et de donner une unité à des documents asisez variés et datant de diverses époques. Il est exact que nous nous étions heurtés à de grandes difficultés pour arriver à donner une certaine unité à notre travail. Des conseils judicieux exprimés sincèrement et amicalement, au cours de francs entretiens, avec $M$. G. Debien, ont contribué à éclairer nos recherches et à former notre jugement. La solidarité des historiens et des amis de l'histoire demeure une réalité bien vivante et infiniment réconfortante.

Du manuscrit dactylographié qui avait été présenté comme thèse complémentaire, nous donnons aujourd'hui sous le titre "Un dossier de plantation (1746-1829), une monographie uniquement centrée sur Saint-Domingue, modeste, mais utile contribution, croyons-nous, à l'histoire de cette ile, où demeurent toujours vivantes la tradition et la culture françaises.

Nos remerciements et notre reconnaissance vont à ceux qui nous ont permis ou procuré l'accès à ces sources dont la découverte nous a causé tant de joie, à ceux qui nous ont guidé et conseillé dans leur utilisation.

Nous tenons à exprimer notre gratitude particulièrement à $M$. G. Debien, à MM. les Professeurs Jacques Godechot, de l'Université de Toulouse, Eugène Revert, de l'Université de Bordeaux qui nous ont fait bénéficier de leur grande compétence.

Il est rare qu'on se trouve devant des documents qui permettent de retracer toute l'histoire d'une plantation depuis ses origines au XVIIe ou au XVIIIe siècle jusqu'à son démembrement au XIXe siècle.

Le fait n'est d'ailleurs pas propre à Saint-Domingue ni même aux Antilles françaises. La Révolution, les incendies, l'humidité et les insectes et plus encore peut-être les partages au moment des 
successions, la négligence des hommes ont dispersé et perdu les documents. Il faut s'y résigner, mais résignation n'est pas découragement.

L'étude que voici sur une des habitations Seguineau sera donc elle aussi fondée sur des documents qui ne sont que des épaves et qui manquent de cohérence. Les archives des plantations Seguineau ont dû être importantes et en bon ordre. Nous n'en avons plus que des miettes: quelques lettres en vue d'un arrangement, des papiers relatifs à un partage familial, un compte courant d'une des habitations avec les frères Terrien de Nantes, chargés de l'administration générale et une partie de leur correspondance avec les nouveaux maîtres d'une des habitations, héritiers des Seguineau, les NavaillesBonnas. Enfin des lettres au sujet de l'établissement des droits à l'indemnité accordée aux anciens propriétaires par la loi de 1826.

Ces documents proviennent de la famille d'Olce de Vic-en-Bigorre, apparentée aux Navailles-Bonnas et sont arrivés par don, en 1949, aux mains de M. l'abbé Gardey, curé de Vic-en-Bigorre qui, avec une obligeance dont nous tenons à le remercier, a bien voulu nous laisser mettre à profit ces vieux papiers.

Nous ne pourrons pas tenter une véritable histoire de cette plantation. Nous ne la verrons que pendant une dizaine d'années. Mais ces années sont des années décisives et comme alors la politique tend à mener la vie économique de Saint-Domingue, les correspondances ne présenteront pas seulement un aperçu du travail - de l'habitation, mais aussi les questions plus générales qui préoccupaient les colons et les hommes d'affaires de la colonie. Nous aurons une bonne idée des discussions politiques de l'heure. Les lettres Terrien qui résument si nettement les opinions d'une partie importante des planteurs méritaient donc à elles seules d'être publices.

L'habitation était plantée en café. Nous avons d'importantes parties d'archives de sucreries. Peu de choses sur les caféières. L'intérêt du compte rendu de 1788, si incomplètes, si incertaines que puissent être les conclusions qu'on en pourra tirer, nous a semblé aussi demander une analyse minutieuse de ce document. Elle éclairera la correspondance des années suivantes. 


\section{LE QUARTIER DE MONTROUY}

Le quartier de Montrouy, où s'établit la plantation Seguineau nous est connu dans son état ancien par la Description de SaintDomingue, de Moreau de Saint-Méry ${ }^{1}$ et par le plan du quartier de l'Artibonite, gravé en 1790 par Philippeaux, ingénieur géographe 2 .

Ce canton portait déjà le nom de Mont-Rouy ou Mont-Rouis, en 1671. Malgré son étendue considérable, cette partie de l'ouest n'avait jamais formé le centre d'une unité paroissiale bien définie, ni un quartier aux limites nettes.

Le Montrouis se trouvait divisé entre les trois paroisses de SaintMarc au Nord, des Verettes, à l'Est et de l'Arcahaye, au Sud. Vers l'ouest était la mer, le golfe de la Gonave. C'est seulement vers 1780,

1. Moreau de Saint-Méry, Description de la partie française de Saint-Domingue (2 vol., Philadelphie, 1797-1798), 2: 216.

2. Localisation de la plantation Seguineau. - Sans la récente découverte au Ministère de la France d'Outre-Mer, de cartes de Saint-Domingue, il n'eât pas été possible de localiser la plantation Seguineau. Jusqu'à ces derniers temps, la Bibliothèque Nationale ne possédait pas de cartes pour les quartiers qui sont au nord du Port-au-Prince: l'Arcahaye, Montrouv, Saint-Marc et les Gonaïves (Bibliothèque Nationale - Département des cartes et plans - trois portefeuilles de plan de SaintDomingue qui viennent du service hydrographique de la Marine).

Egalement de grandes difficultés se présentaient pour essayer de situer l'habitation grâce à la carte moderne de la République d'Haîti au $1 / 400.000^{\circ}$ publiée par la direction générale des travaux publics à Port-au-Prince, en juillet 1928. Cependant par différents papiers, nous savions que la plantation des frères Seguineau n'était pas très éloignée de l'habitation de Lugé. Or la carte précédente indiquait à 4 ou 5 kilomètres au nord de l'embouchure de la rivière de Montrouis le nom: Delugé, à droite de la route du Port-au-Prince à Saint-Marc, à une douzaine de kilomètres de Saint-Marc. De plus, dans un article (Rev.d'Hist. des Colonies, no 4, (1933): 219-220240), M. Hervé du Halgouët (Inventaire d'une habitation à Saint-Domingue) parle de "Jean Paqué", comte de Lugé, décédé le 11 novembre 1788", et note comme expert "Lanzac voisin". Comme dans les papiers de la famille Navaille figure un projet d'achat de l'habitation de Lanzac qui était une sucrerie, nous pensions les deux plantations voisines et nous supposions alors la plantation Seguineau plus haut vers les mornes et descendant jusqu'à la Rivière de Montrouy.

Les cartes récemment découvertes, notamment la carte - B 5 - "Plan du quartier de l'Artibonite, isle de Saint-Domingue, renfermant les paroisses de SaintMarc, de la Petite Rivière et des Verettes, dédié à M. le Cto d'Agoult - Paris, Philippeaux 1790 - Gravures coloriées - armoiries" - confirme nos précédentes suppositions et permet de situer avec précision l'habitation des frères Seguineau.

Du haut des mornes du Fond-Baptiste, l'habitation descend vers la rivière de Montrouy, forme un grand rectangle, limité au sud par les mornes; au nord, par le cours de la rivière de Montrouy, au delà de laquelle, toujours au nord se trouvent la plantation Gresseau, Bachette et Olynette, à l'ouest, la plantation Finchinat; à l'est, la caféière E. Farette.

Dans ce quartier de plantation de café, la plantation Seguineau apparaît comme la plus importante par sa superficie. 
et après des années de récriminations des colons, qu'on forma le projet de constituer ce quartier en paroisse sous le nom de Vaivre, emprunté à l'intendant du moment, à Saint-Domingue, mais en 1790, Philippeaux nous montre que ce projet n'était pas sorti des bureaux du Port-au-Prince.

La rivière du Montrouy et ses petits affluents traversaient la région. A Saint-Domingue, tout ruisseau dès qu'il était permanent, ou à peu près, se voyait décoré du grand nom de rivière. Celle-ci courait pendant huit lieues, mais ses rives n'avaient vraiment d'eau qu'aux jours de pluie. Les autres cours d'eau servaient à l'arrosement de sept sucreries et à la marche de leurs moulins à cannes.

Dans ce pays, au relief si profondément raviné et compartimenté, le climat de la région de l'Artibonite était connu pour sa sécheresse. 1782 et 1783 avaient été presque sans eau. "C'est vers le Mont-Rouis, dit Moreau de Saint-Méry, que la privation des pluies est cruellement sentie. Lorsque j'y passais, en avril 1783, on n'y avait reçu depuis vingt mois que trois grains de pluie. Il n'en tombe pas d'ordinaire avant le mois de juin et quelquefois celui de juillet. On y plante cependant des cannes, sans égard à l'époque des roulaisons, parce que l'unique nourriture des animaux ce sont les têtes de ces mêmes cannes à sucre $^{3}$."

La sécheresse aidera aussi au développement des insectes. "Les pucerons désolent le Montrouis, depuis plus de quarante ans; tout en est couvert jusqu'aux haies." Le manque d'eau explique que peu de sucreries avaient pu s'installer et que toutes dépendaient directement de ruisseaux captés. Il ne faut pas aller chercher ailleurs le développement tardif du quartier tout en mornes. L'introduction de la culture du café, son développement dans ce quartier fut la base d'une nouvelle richesse et d'un rapide peuplement.

Il $\mathbf{y}$ avait une vingtaine d'années que Nolivos avait planté des caféiers dans la colonie à Léogane ${ }^{5}$.

Le café était cultivé dès 1738 dans le Nord, dans les mornes du quartier du Dondon ${ }^{6}$.
3. Moreau de Saint-Méry, Description... 2: 218, 219.
4. Ibid., 2: 220.
5. R.P. Cabon, Histoire d'Haïti (4 vol., Port-au-Prince, s.d.).
6. Ibid., 2: 490 . 
C'est à partir de 1743 que le canton de Montrouis comme toute la zone des mornes intérieurs depuis l'Artibonite jusqu'au fond du Mirebalais et à la frontière espagnole s'ouvrira à la nouvelle culture $^{7}$.

En 1745, les quatre frères Seguineau obtiennent une large concession pour la culture du café. Ce n'est pas encore la culture en grand; on prend rang, on fixe des droits, on essaie les sols; on s'installe çà et là loin de la plaine aux cannes. L'essor viendra après et de la réunion de ces essais de début, de la concentration de plusieurs concessions primitives naitront de grandes plantations caféières.

Les terrains de la plantation Seguineau seront au contraire très grands dès le début, parce que les quatre frères jumelant leurs demandes de concession ont su en obtenir une qui représente juxtaposée en un immense carré, de 1.200 toises environ de côté, leurs droits indivis.

Moreau de Saint-Méry qui paraît connaître assez mal tous ces quartiers de mornes, a pris soin de nous rapporter l'opinion commune des colons sur la valeur des différentes zones de hauteurs qui, du bord de la mer s'élevaient vers la partie espagnole. "Les mornets qui bordent immédiatement les plaines, nous dit-il", sont, en général, mauvais, arides et peu susceptibles de cultures. Quelques habitants y élèvent des animaux. On y récolte un peu de coton, des vivres de terre $^{9}$ et des légumes médiocres. Les montagnes élevées sont dans leur face tournées vers la mer de la même nature que ces mornets. On pourrait dire que jamais bordure plus sombre n'a été donnée a un tableau plus gai."

Mais tout autres sont les hauteurs du Fond-Baptiste où se situe la concession Seguineau. En effet, "dans l'autre face, les montagnes sont au contraire fraiches et fertiles, comme le prouvent de magnifiques caféteries et notamment celle des deux cantons si célèbres des Matheux et du Fond-Baptiste. Comme le premier de ces deux endroits est plus chaud que l'autre, la maturité du café y arrive plus

7. C'est vers 1743-1745 que la caféière Maulevrier - dont M. Debien prépare l'étude - offre une série d'une vingtaine de concessions, toutes de ces années, toutes pour hattes et caféières.

8. Moreau de Saint-Méry, Description... 2: 254, 256.

9. Ignanes, patates, manioc. 
tôt et la récolte y est terminée avant les pluies. Leur récolte commune est entre 3 et 4 millions de livres de cafe ${ }^{10}$."

"Ces cantons, ajoute Moreau de Saint-Méry, font partie des mornes du Mont-Roui et n'ont été concédés que vers $1743-1744 \ldots$ Dans la même partie montagneuse, se trouvent le canton des Délices, ceux de la Nouvelle-Saintonge, de la Nouvelle-Gascogne, de la Nouvelle-Rochelle ${ }^{11}$."

Une trentaine d'années après, le quartier était assez peuplé pour être pourvu d'une police; le bourg du Montrouis n'était encore qu'un petit groupe de 14 cases. La richesse d'un quartier ne se voyait pas à la splendeur des monuments de son bourg. Un autre indice nous montre à la fois le quartier sortant de son isolement et s'enrichissant: "Dans les dernières années de la guerre (donc entre 1780 et 1782), M. Chanlotte (sans doute un mulâtre libre) habitant du Mont-Roui, fit l'entreprise de louer des chaises et des chevaux pour aller de Saint-Marc à l'Arcahaye... ${ }^{12}$ "

D'après la carte $\mathrm{B} 5$, des archives du Ministère de la France d'Outre-Mer. (Plan du quartier de l'Artibonite, isle de Saint-Domingue, renfermant les paroisses de Saint-Marc, de la Petite-Rivière et des Verettes, dédiée à M. le Cte d'Agoult - Paris Philippeaux, 1790 - Gravures coloriées - armoiries.)

Dans la partie montagneuse du Fond-Baptiste "on n'en finirait pas, dit Moreau de Saint-Méry ${ }^{13}$ si on voulait nommer tous les cantons... presque chaque habitant en fait un de son habitation." Autrement dit, la jeunesse même de la toponymie exprimait l'arrivée récente de l'homme et le caractère encore dispersé de son effort agricole.

Jusqu'à la Révolution, en effet, ce quartier ne sera pas un lieu de très vives disputes, pour obtenir de la terre. L'habitation Seguineau, en 1790, est encore bornée par des terrains appartenant à des mulâtres libres, qui ont dû être là comme en tant d'autres

10. Moreau de Saint-Méry, Description... 2: 255.

11. Ibid., 2: 254, 255.

12. Ibid.

13. Ibid., 2: 216, 217. 
quartiers, les pionniers du déboisement et de la vie agricole. Ailleurs, ces premiers occupants ont vendu leurs droits. Ici, on voit toujours à l'ouest de l'habitation Seguineau la concession de Pinchinat, mulâtre libre et sur la rive droite de la rivière du Montrouis, la concession de Gresseau, autre mulâtre libre.

Le cadre humain de la concession Seguineau, fait rare dans l'histoire de Saint-Domingue, paraît avoir peu changé, de 1745 a 1790.

CHAPITRE PREMLER

DEUX FAMILLES DE PLANTEURS ET DE PROPRIÉTAIRES COLONIAUX:

\section{LES SEGUINEAU ET LES NAVAILLES-BONNAS}

\section{LES SEGUINEAU.-}

Les Seguineau étaient originaires de Saintonge, de "Bourgen-Méchet"14. Ils appartenaient à une famille de bourgeois et de négociants qui devait venir s'établir à Bordeaux au 18e siècle, rue des Petites-Carmélites, paroisse Saint-Michel, puis paroisse SaintRémy, cours du Chapeau-Rouge. Un frère habitait rue Dorade, no 4. Déjà au $17 \mathrm{e}$ siècle, on trouve des Séguineau à La Rochelle. Mais Bordeaux fut pour eux la ville où s'exerça l'attraction de l'Amérique. Ils y possédaient un hôtel de famille et dans la proche banlieue le domaine de Lognac.

Les membres de cette nombreuse famille ont dû jouer un rôle éminent dans la société bordelaise du XVIIIe siècle, car les Seguineau étaient gens d'assez large surface. La généalogie ${ }^{15}$ donne une liste impressionnante de Seguineau à cette époque. Il y avait cinq garçons et une fille. Notons Nicolas Seguineau qui meurt avant 1771. Pierre-Louis Seguineau, propriétaire à Saint-Domingue, sans alliance, qui teste le 6 mars 1786 devant Me Barberat; Jean Seguineau également propriétaire à Saint-Domingue, teste dans l'île le

14. Bourg-en-Méchers (Charente-Maritime).

15. Généalogie fournie par M. le Sous-Archiviste de la Gironde. 
30 octobre 1763. Des quatre frères partis en 1745 aux Iles, seul Jean Seguineau devait retourner en France et mourir au Château de Lognac en 1772. Il fut inhumé dans l'église Sainte-Eulalie de Bordeaux, le 25 juillet 1772 . Quant aux deux autres frères, Louis Seguineau, propriétaire à Saint-Domingue, meurt au quartier de Montrouy le 5 janvier 1771. Il avait testé le ler décembre 1764 devant Grandmaison, notaire à Saint-Marc.

Le dernier frère, Pierre Seguineau, jeune, avait acquis une charge de secrétaire. du roi et il fut annobli. Il est seigneur du fief des Carmes à Pian en Médoc, à la date du 2 février $1783^{16}$, des maisons nobles du Luc et Andride, paroisse de Pian, en Médoc et de Blanquefort, de la maison noble de Légune à Ludon-Médoc.

Il y avait également une sœur, Marie Seguineau, mariée à un bourgeois, M. Comte et dont la fille Françoise Comte épousera François Maillet, lieutenant de la Fauconnerie du Roi. Ils passeront tous deux à Saínt-Domingue, où Maillat achètera une plantation ${ }^{17}$. François Maillat, neveu des Seguineau, sera en même temps gérant de l'habitation Seguineau.

Les quatre frères Seguineau: Pierre Louis Seguineau, dit l'aîné, Jean Seguineau, Louis Seguineau et Pierre Seguineau, dit le jeune, ayant obtenu en 1745, une concession à Saint-Domingue, au quartier de Montrouy et à la Nouvelle-Saintonge, paroisse Saint-Pierrede-l'Arcahaye, dépendance du Port-au-Prince, tailleront à même le paysage un immense rectangle au pied des mornes jusqu'à la rivière Montrouy et exploiteront en commun ce terrain.

Les Seguineau semblent être venus du négoce à la culture. Peutêtre même ont-ils joint la culture au commerce. C'est probable puisqu'ils avaient une maison de commerce avec des entrepôts, des magasins et des maisons à Saint-Marc et au Port-au-Prince. Il faut croire qu'ils ont été marchands avant d'être planteurs. C'était d'ailleurs' la règle. Cette classe de petite bourgeoisie marchande a eu une grande part dans la colonisation des Antilles aux XVIIe et XVIIIe siècles.

16. La mention figure dans un acte du notaire Rauzan (2 février 1783) - Archives de la Gironde, série E.

17. Maillat avait une plantation au Fond-Baptiste, en 1778, dite Maillat et Roche. - Archives de la Vienne En 1970. 
Il est très fréquent que l'on sache peu de choses sur les origines et les premières années des plantations. En 1745, on se mettait au café dans ces hauteurs de l'Arcahaye. Mais ce qui est rare alors, c'est l'installation de grandes plantations en café, telle celle des Seguineau. C'était apparemment une des premières du quartier ${ }^{18}$. A l'origine, la concession obtenue par les frères Seguineau resta dans l'indivision et fut exploitée en commun. Par suite d'héritages et d'arrangements de famille, elle devait passer aux mains de la famille de Navailles.

Ces Seguineau avaient d'autres plantations. L'ensemble de leurs biens, d'après l'État des indemnités, comprenait des caféières, des cotonneraies; une petite caféière, au Fond-des-Nègres, dans le Sud, estimée 30.000 livres; à l'Arcahaye, à une cinquantaine de kilomètres au Nord de Port-au-Prince, une autre caféière et des maisons à Saint-Marc. L'ensemble était estimé 550.000 livres de France, soit 750.000 livres de Saint-Domingue. La seule caféière du Fond-Baptiste, dite Seguineau frères, était estimée à elle seule 447.790 livres d'après l'Etat de 1829 , et d'après les papiers Navailles, 498.842 francs 67 centimes $^{19}$.

Parmi les bourgeois et négociants nombreux qui possédaient aux Iles, les Seguineau occupent une place importante.

\section{LES NAVAILLES.-}

Le berceau de la famille Navailles était Larreule, en Bigorre ${ }^{20}$. Les Navailles étaient de gros propriétaires terriens qui ne cesseront

18. On fait, en effet, peu de café à Saint-Domingue avant 1765. La date du début de la culture du café diffère suivant les auteurs. Ainsi: James, Les Jacobins Noirs (éd. Gallimard, 1949), écrit, p. 4: “...en 1734, les colons commencèrent à cultiver le café..." Dans les Annales - Ec. Soc. civ. no 4, (oct. décembre 1949), p. 494, $M$. Braudel souligne: “ce n'est qu'd partir de 1723 , que les caféiers s'introduiront dans les Antilles, ainsi a La Martinique et à Saint-Domingue où ils se substituent aux cacaoyers en 1727-1728..."

M. G. Martin, enfin dans son ouvrage, L’ère des négriers, écrit, p. 138: “...très intéressant est aussi la progression du café. Il n'apparait qu'à partir de 1790, dans les déclarations de retour - d'abord très rare, il figure presque a égalité avec le sucre dans celles de la fin du règne de Louis XV..." De toute façon, 1743-1745 marque au Montrouy le début de la culture du café.

19. Etat des indemnites - Archives Nationales. - Ministère des Finances. Etat détaillé des liquidations opérées par la Commission chargée de répartir l'indemnité attribuée aux anciens colons de Saint-Domingue (6 vol., Paris 1822-1833). Bibliot. Nation. Lf $158 / 41$.

20. Larreule-en-Bigorre, à $14 \mathrm{~km}$. au nord de Tarbes. 
de s'intéresser à leurs terres provinciales et à leurs terres coloniales, car par mariage ou par héritage, ils ajouteront à leurs biens en France des biens coloniaux importants. C'est le type même de la famille de grands propriétaires coloniaux résidant en France et dont l'absentéisme sera à juste titre si vivement critiqué par la suite.

Ils n'étaient d'ailleurs pas seuls dans la région à posséder aux Antilles. Bigourdans ou Béarnais tout proches, ils sont nombreux ceux qui ont eu des biens à Saint-Domingue. Des Béarnais furent propriétaires ou colons aux Iles. Certains d'entre eux étaient des parlementaires, d'autres furent simplement colons. Citons les Duplas, les Charritte. Parmi les Bigourdans, les Navailles-Bonnas, Davezac de Castéra, Charles Maigné de Saint-Martin ${ }^{21}$. Les uns et les autres, de près ou de loin, s'intéressent au développement de l'île.

Parmi les Bigourdans, les Navailles apparaissent au premier plan.

Joseph de Navailles-Bonnas était en 1752 lieutenant d'une compagnie détachée de la Marine au quartier du Cul de Sac. Il fut aide-major des troupes de Saint-Domingue, de 1770 à $1778^{22}$. Il avait servi en France avant de passer à Saint-Domingue. Il est major du quartier de Mirebalais en novembre 1770. Ce quartier était aussi un quartier de plantation de café mis en valeur après 1763 et Joseph de Navailles avait une plantation dans ce quartier. Cette habitation se situe le long de la rivière de l'Artibonite, au lieudit la Ravine à couleuvres, dépendance du Petit-Fond; elle comprenait 144 carreaux de terre. Elle était bornée au sud par la rivière de l'Artibonite, à l'ouest, par la plantation Dammond et au nord et à l'est, par la ligne de démarcation des limites françaises et espagnoles. Cette concession avait été accordée à Navailles, le 16 octobre 1776, par "Messieurs d'Ennery et de Vaivre, général et intendant de cette colonie". La plantation fut arpentée par un sieur Lecoudray, arpenteur du roi, le 20 janvier 1777. Le procureur en fut un nommé Leydier.

Joseph de Navailles-Bonnas quitte Saint-Domingue, en 1778, pour raison de santé, semble-t-il, laissant son habitation "à titre de

21. Dans les Hautes Pyrénées, certains lieuxdits rappellent cet engouement ansien pour l'Amérique, Ainsi le lieudit: "l'Amérique, entre Bordères-sur-Echez et Ibos - et le Tour des Américains, (promenade aux environs de Vic), à Vic-Bigorre.

22. Moreau de Saint-Méry, Description... 2: 241. 
bail à ferme", pour sept ans, c'est-à-dire du 1er novembre 1778 au 1er novembre 1785. Il meurt à Larreule, où il s'était retiré, le 29 mai 1781 et est inhumé dans le petit cimetière de son village, où de nos jours sa tombe existe encore ${ }^{23}$.

Son frère, Jean-Baptiste de Navailles, seigneur de Bonnas, était conseiller au Parlement de Pau. Il avait épousé le 11 juin $1770^{24}$, Françoise Claire Seguineau, "fille de feu Nicolas Seguineau et de vivante demoiselle Françoise Maillat, demeurant à Bourg-deMéchers."

Cette alliance avec les Seguineau est une alliance avec la roture. Les raisons du mariage furent-elles intéressées? Il est délicat de se prononcer sur ce point. Navailles-Bonnas avait une situation personnelle importante, mais les Seguineau tenaient une grande place dans le négoce bordelais. De plus, le père de Françoise Claire Seguineau était mort avant le mariage de sa fille. Ses frères n'avaient pas d'héritiers directs; en cas de décès de l'un d'eux, leur nièce devait hériter de leurs biens. Ces biens paraissent avoir été conséquents, même si l'on ne compte que les biens patrimoniaux de France.

Dans le contrat de mariage du 11 juin 1770, Jean Seguineau et Pierre Seguineau s'engagent à payer à Jean Baptiste de NavaillesBonnas l'intérêt, l'un de $\mathbf{3 0 . 0 0 0}$ livres, l'autre de $\mathbf{5 0 . 0 0 0}$ livres, à $5 \%$. Cette somme constituait la dot de leur nièce. Aussi le mariage de Navailles ne fut peut-être pas exempt de calcul. Toujours est-il que, par son mariage, il va désormais appartenir à cette classe si mal connue des grands propriétaires coloniaux résidant en France. Cette union va lui permettre d'augmenter son capital. Mais - et c'est regrettable - les documents sont trop fragmentaires pour savoir si les capitaux venus de Saint-Domingue ont ;aidé à l'achat de terres en France, ont permis une vie luxueuse avec un train de maison plus important ou si des capitaux venus de France n'ont pas contribué à relever la plantation.

Navailles-Bonnas mènera une existence aisée, partageant son temps entre sa charge de conseiller au Parlement de Pau et la surveillance de ses terres en Bigorre. Il séjournera surtout à l'Hôtel

23. Dans les papiers de famille figure son brevet de lieutenant-colonel signé de la main de Louis XV et de Sartines.

24. Contrat de mariage du 11 juin 1770 - Papiers Navailles-Bonnas. 
Seguineau, à Bordeaux. Il possède de hautes relations à Versailles, si nous en croyons une lettre de $M$. de Malesherbes, ministre du roi. En effet, en 1774, Navailles-Bonnas adressait ses vœux à M. de Malesherbes et sollicitait des lettres de noblesse pour ses oncles Seguineau et la croix de Saint-Louis pour le neveu de Maillat.

(à Versailles, le 19 janvier 1776$)^{25}$.

"...les grâces que vous sollicitez en leur faveur ne dépendent pas de moi. M. Maillat ayant servi dans les colonies, c'est à M. de Sartines à faire valoir ses services pour obtenir la Croix de SaintLouis. La ville de Bordeaux où demeure M. J. Seguineau est du département de $M$. Bertin et c'est à lui qu'ils peuvent s'adresser pour les lettres de noblesse qu'ils voudraient avoir. Mais je dois vous prévenir que le Roy ne les accorde qu'avec la plus grande difficulté."

A Bordeaux également, les relations de Navailles-Bonnas devaient être étendues, notamment dans le milieu des propriétaires coloniaux. En 1789, il sera membre du club des colons de Bordeaux et ce club était en relations avec le club des colons de Paris qui cherchèrent à faire face à la Révolution ${ }^{26}$.

A la mort de J.-B. de Navailles-Bonnas, son fils Maximilien vivra quelques années encore à Larreule, auprès de sa mère, Françoise Claire Seguineau. Quand sa mère mourra, en 1808, Maximilien qui est le préféré de ses trois enfants, recevra d'elle, par testament, à titre de préciput et avantage, tous ses biens meubles et immeubles.

Maximilien de Navailles-Bonnas a fait les campagnes de l'Empire. Chevalier de la Légion d'Honneur, en 1813, capitaine dans la Légion des Hautes-Pyrénées, en garnison à Carcassonne, en 1818, il prend sa retraite vers 1820 , pour se retirer à Larreule et vivre du revenu de ses terres. Il a servi l'Empereur, puis les Bourbons. Il meurt à Larreule, en 1836, après avoir touché péniblement la dérisoire indemnité, accordée aux anciens propriétaires de Saint-Domingue, laissant ses biens, à son neveu d'Olce.

25. Correspondance Navailles-Bonnas.

26. Archives nationales, Dxxv 86. 
Grâce à divers arrangements de familles, les Navailles vont, au cours du XVIIIe siècle, devenir propriétaires de la plantation Seguineau.

Le 1er septembre 1764, Louis Seguineau teste devant Grandmaison, notaire à Saint-Marc, en faveur de Pierre Seguineau le jeune, son frère, habitant au quartier de Montrouy. Il l'institue son légataire universel. Louis Seguineau devait mourir sept ans plus tard, le 5 janvier 1771. La plantation reste aux mains des deux frères survivants: Pierre Seguineau aîné et Pierre Seguineau jeune qui a en plus la part de son frère décédé Louis. Tous deux auront également la part de leur autre frère, Jean Seguineau qui avait testé à Saint-Domingue, le 30 octobre 1753 et était mort au château de Lognac, le 25 juillet 1772.

Le 29 mai 1773, apparait la première transaction entre les Seguineau et les Navailles-Bonnas, transaction entre les deux frères survivants et leur nièce Françoise Claire Seguineau, épouse de Jean Baptiste de Navailles-Bonnas. Il s'agit de prévenir les contestations relatives à l'exécution du testament de Jean Seguineau, frère de Seguineau aîné et de Seguineau jeune et oncle paternel de Mme de Navailles.

L'essentiel de la transaction ${ }^{27}$ porte sur les conditions où vont se faire le partage des revenus. En somme, on restera dans l'indivision avec un gérant que connaissent les Seguineau, leur neveu Maillat. Les frères Seguineau renoncent au legs universel fait en leur faveur par leur frère et ils consentent que leur nièce, présomptive héritière pour un cinquième, jouisse et dispose de la cinquième partie de la succession.

Par cette transaction de 1773 , les Navailles commencent à être intéressés dans la gestion de l'habitation. Il faut attendre encore quelques années pour que nous ayons une idée exacte de cette plantation. De 1745 à 1788, nous n'avons rien sur l'exploitation de cette caféière. Nous savons seulement qu'après 1770 et le départ du dernier Seguineau, la caféière a eu pour gérant François Maillat, leur neveu.

27. Papiers Navailles-Bonnas. 
Maillat, ancien lieutenant de la Fauconnerie du Roi, régira l'habitation pendant une quinzaine d'années; il sera remplacé par Guchen, puis par Girodon. Les frères Terrien, originaires de Nantes, seront les procureurs. Le compte de gestion de 1788 est signé par les frères Terrien.

Enfin Pierre Seguineau, oncle paternel de Madame de Navailles et dernier survivant, teste, le 18 mai 1789 , devant Me Barberet, notaire à Bordeaux, en faveur de sa nièce à qui il lègue son habitation avec toutes ses dépendances et la moittié d'une maison. Il meurt la même année.

Ainsi, à la veille de la Révolution, les Navailles-Bonnas sont propriétaires de l'habitation Seguineau. Il se trouve que pour l'une des dernières années normales de la plantation, pour 1788, nous avons un compte général de la gestion. Il est naturellement encore adressé à l'aîné des Seguineau. C'est ce compte de gestion qui va nous donner des renseignements intéressants sur la plantation au XVIIIe siècle.

\section{CHAPITRE DEUXIÈME}

\section{UN COMPTE DE GESTION DE CAFEIERE (1788) ${ }^{28}$}

L'absence du propriétaire, le système des procureurs et des gérants est à l'origine de la comptabilité des plantations. Il était nécessaire pour le propriétaire résidant en France de connaître le revenu exact de ses terres. Il fallait envoyer les bénéfices en France s'il y avait des bénéfices et dans le cas contraire, étudier le moyen de payer les dettes. Les ordonnances du 3 décembre 1764 et du 23 décembre 1765 avaient réglementé la tenue des livres d'habitation. Les procureurs et les gérants tenaient plusieurs sortes de livres dont le grand livre des plantations où figuraient le détail des tra-

28. 1788 - Compte de gestion - Habitation de M. Seguineau aîné. - Papiers Navailles-Bonnas. 
vaux, l'état des noirs, les recettes et les dépenses. Les gérants rendaient leurs comptes tous les ans. Un relevé des comptes de l'année était adressé au propriétaire en France par le procureur. Le compte de gestion se divisait en doit et avoir, la balance du compte était faite au dernier feuillet et il était possible de voir s'il y avait accroissement ou diminution des revenus ou dettes.

Ces comptes étaient destinés à surveiller l'activité de la plantation et des gérants. Mais tant vaut l'homme qui rédige les comptes, tant vaut l'honnêteté de l'exposé. Et très souvent ces pièces n'avaient pour but que de justifier les fortes dépenses, cacher des malhonnêtetés ou couvrir une mauvaise administration.

Le compte de gestion de la caféière Seguineau-Navailles ne répond qu'imparfaitement à notre curiosité. De nombreuses questions qui se posent resteront sans réponse. Nous ne pourrons pas notamment donner le rapport précis de l'habitation en café. Néanmoins, il présente un réel intérêt étant donné la rareté de ces documents, car presque tout manque de la comptabilité des grandes caféières.

Les rares comptes de gestion conservés ne sont souvent qu'un extrait du livre de caisse, un simple relevé des recettes et des débours. D'autre part, il serait intéressant d'avoir des comptes de plusieurs années pour bien saisir l'évolution de la plantation et nous ne possédons qu'un seul compte de gestion.

Même dans un compte exact, il n'est pas toujours facile de voir s'il y avait accroissement ou diminution de valeur. Au surplus, certaines parties du compte demeurent obscures. S'agit-il de dépenses toujours urgentes et productives? Quelques renseignements donnés par la correspondance des procureurs et de leur maitre n'apporteront pas une grande clarté sur les dettes et les revenus nets. Il faut savoir se contenter des documents en notre possession et les utiliser au mieux.

Les procureurs de la plantation, en 1788, sont les frères Terrien, soit Terrien aîné, ou Terrien jeune. Ils conserveront la direction de la caféière de 1788 à 1797. En 1794, Terrien aîné passera en Amérique du Nord, laissant la charge de l'habitation à son frère.

Certaines parties de ce compte, rédigé par les frères Terrien demeureront incomplètes, car ce n'est pas exactement un compte de gestion que nous avons rendu par les procureurs gérants, les frères 
Terrien. Pour être tout à fait précis, il faut dire que c'était à la maison Terrien frères de Nantes que la direction générale de l'habitation était confiée. Cette maison de commerce était en compte avec les habitations des Seguineau, achetant leurs cafés, ou une partie de leurs cafés, et fournissant certains approvisionnements. Ici, ils rendent un compte de ce qu'ils ont dépensé non seulement pour la marche de l'habitation, mais aussi pour l'entretien des maisons et magasins que les Seguineau avaient à Saint-Marc, pour la marche de l'habitation et de ce qu'ils ont acheté à l'habitation. Ce n'est donc pas tout à fait un compte de gestion. D'autre part, la non distinction des dépenses pour Saint-Marc et des dépenses pour la plantation crée une certaine confusion et Seguineau aîné ne paie que $1 / 4$ des frais parce que beaucoup de choses restent dans l'indivision.

\section{LE CAPITAL D'EXPLOITATION.-}

Dans toute estimation du capital d'exploitation, il faut faire entrer les esclaves. Leur nombre - 150 - est ici important pour caféière et c'est la plus grosse part du capital. Les bâtiments d'une caféière étaient moins importants que ceux d'une sucrerie, les bestiaux ne servaient pas au travail, mais au transport et nous ignorons les pertes d'animaux.

Dans le cas présent, le capital d'exploitation peut être évalué à 355.200 livres, pour la main-d'œuvre, plus 200.000 livres pour les bâtiments et la valeur des terres, soit au total 555.200 livres ou 700.000 livres environ de biens coloniaux.

Analysons d'abord le compte débiteur.

Le solde de l'exercice 1787, arrêté au 30 décembre est de 3.848 livres $14 \mathrm{~s} .11 \mathrm{~d}$. Ce n'est pas un compte de l'habitation seule que nous avons, mais des intérêts des Seguineau à Saint-Domingue, puisqu'il est question de dépenses pour réparer une petite maison à Saint-Marc.

DÉPENSES et RECETTES. -

I. - LES DÉPENSES. - Il est done nécessaire d'étudier en premier lieu les dépenses étrangères à l'habitation proprement dite. Ces dépenses sont relatives aux frais d'octroi, à des réparations, à l'établissement de barrières, à l'étalonnage des poids du Montrouy. 
Deppenses étrangères à l'habitation proprement dite:

- Reparations à la maison de Saint-Marc

- Octrois pour la maison de Saint-Marc, de 1784 à 1787.

- Octroi pour une autre maison à St-Mare.

- Etablissement d'une barrière à la maison de de Saint-Marc. . . . . . . . . . . . . . . . . ....

- Fournitures pour réparer la maison de SaintMare.

- Etalonnage des poids.

- Fournitures pour la petite maison de St-Marc.

- Fournitures pour la petite maison de St-Mare.

Total

6 liv.

$187-10 \mathrm{s.}$

$160-8$ s. 4 d.

$100-$

$46-85 \mathrm{~s}$.

7 - $6 \mathrm{~s} . \quad 3 \mathrm{~d}$.

$36-15$ s. $2 \mathrm{~d}$.

$548-4$ s. 9 d.

Les frais d'exploitation consistent dans:

- Les transports de café (frêt pour les transports).. 1.666 liv. $53 \mathrm{s.} 28 \mathrm{~d}$.

— Frêt de 5 balles et de 2 boucauts........... 52 - $10 \mathrm{s.}$

- Frais pour moulins à maïs............. 4 -

- Frêt de 18 barriques de vin à $70 l$. le tonneau. .. . 315 -

- Colliers à piler le café.................. 61 -

- Frais de magasinage.................. 689 -

- Fournitures pour le magasin............. 609 Ajoutons:

- le charroi du café au magasin..............

- et une commission de $4 \%$ (pour les frères Terrien) sur $95.706 l .13$ s. montant du produit du café, soit.

$74-$

17 s. $6 \mathrm{~d}$. 55 s. $12 \mathrm{~d}$.

Ce qui donne un total de.

$\begin{array}{lll}3.828- & 5 \text { s. } & 3 \mathrm{~d} . \\ 7.305-9 \text { s. } & 9 \mathrm{~d} .\end{array}$

Les frais d'entretien vont:

- aux ouvriers qui réparent, à l'achat de matériaux, et de pots pour l'hôpital..............

- au raccommodage des fléax...............

- aux façons d'essentes..................

- à l'achat de planches, clous.................

- à l'achat de planches pour remplacer les fonds des boucauts..........................

- Ferrements, chaux, carreaux, etc........... 154 -

— barils de sel. .......................

- 4 paires de souliers pour le gérant.

10 liv. 13 s. 9 d. $14-11 \mathrm{~s} . \quad 3 \mathrm{~d}$. 459 - 7 s. 9 d. $100-$
48

$33-$

17 s. $6 \mathrm{~d}$.

$2.432-10 \mathrm{~s}$. 
Dans les frais de main-d'œuvre, on peut comprendre:

- l'achat de noirs (6 têtes de noirs, en 1788 à 2.400 liv.)......................... 14.400 liv.

— L'achat de couvertures de laine........... 66 -

- Rechange pour les 6 nouveaux nègres.......... 84 - $76 \mathrm{~s}$.

- Enfin des gratifications diverses (étrennes aux commandeurs, aux négresses, etc...)......... $106-15 \mathrm{s.} 10 \mathrm{~d}$.

soit un total de frais de main-d'œuvre, de:..14.660 - 11 s. $10 \mathrm{~d}$.

Les impôts, droits municipaux s'elèvent pour les 148 têtes de noirs (3 liv. par têtes à l'Arcahaye)

à........................ 444 liv.

Dans les dépenses entrent également:

Les frais de gestion, aux gérants, economes, chirurgiens:

- Appointements du gérant Bourron. .......... 1.250 -

- Appointement du gérant Guchen...........6.6.000 -

- Appointement du chirurgien Roulez......... 1.500 -

- Etrennes aux commandeurs............. 33 -

Ajoutons ce que l'habitation doit à Bourron....... 4.349- $6 \mathrm{s.} \quad 6 \mathrm{~d}$.

et le paiement de quelques dettes pour une somme

de:.............................. $142-8$ s. $3 \mathrm{~d}$.

ce qui nous donne un total de..........13.274 liv. $14 \mathrm{s.} 9 \mathrm{~d}$.

Les frais généraux se réduisent:

- aux abonnements aux Affiches ${ }^{29} \ldots \ldots \ldots \ldots \ldots 16-10 \mathrm{~s}$.

- à la participation de l'habitation aux dépenses pour l'église de l'Arcahaye. .............. 1.163 - 18 s. 6 d.

— à l'achat de fusils...................... 24 - $15 \mathrm{s.}$

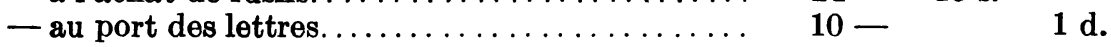

soit.......................... 1.215 liv. $3 \mathrm{s.} 7 \mathrm{~d}$.

Les frais de procès ${ }^{30}$ de succession, font aussi partie du débit. Ils se chiffrent à.$\ldots \ldots \ldots \ldots \ldots \ldots \ldots \ldots \ldots \ldots \ldots \ldots \ldots \ldots .257$ liv. 2 s. 6 d.

Notons que Seguineau, en 1788, ne paie que le quart des frais, parce que beaucoup de choses restent dans l'indi-

29. En 1788, l'habitation est abonnée aux "Affiches américaines", de l'imprimeur Mozard de Port-au-Prince. Le prix de l'abonnement est de 66 livres par an.

30. Les procès étaient un fléau à Saint-Domingue et Saint-Domingue a souvent été comparé à un pays de chicane comme la Normandie. 
vision et ce qui complique le compte c'est qu'il prend parfois le tiers des frais généraux ou des recettes, parfois la moitié. Enfin, dans tous les comptes, il y a au débit les expéditions de denrées et aux recettes les denrées estimées ou vendues sur place. C'est probablement une manière de compter en partie double et cela tend à nous masquer les véritables bénéfices, si nous ne prenons pas la précaution de retrancher du débit les expéditions de denrées pour arrêter la balance du compte.

Les expéditions de denrées figurent au débit consistent en 56.138 milliers de café montant à: 63.925 liv. $6 \mathrm{~s}$.

Une récapitulation de ce débit nous donne une idée plus nette des dépenses de la plantation:

- Dépenses étrangères à l'habitation proprement

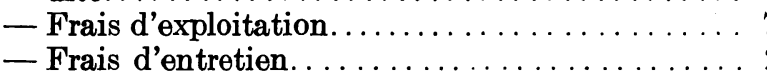

- Frais d'entretien......... 554 liv. $\quad 4$ s. $9 \mathrm{~d}$.

- Frais de main-d'œuvre. . . . . . . . . . . . . . . . . 14.660 -

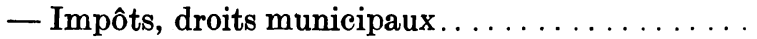

$9 \mathrm{~s}$. $9 \mathrm{~d}$.

— Frais de gestion. . . . . . . . . . . . . . . . . . . . 13.274 -

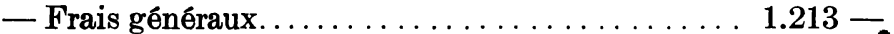

- Frais de procès, de succession. $63.925-6 \mathrm{~s}$.

Le débit total de M. Seguineau s'établit donc à la somme de 105.060 livres, 22 s. 10 d.

Le chiffre le plus élevé des dépenses réside dans les frais de main-d'œuvre, viennent ensuite les frais de gestion et d'exploitation. Les impôts (droits municipaux) sont faibles parce que le principal impôt était les droits de sortie.

Il ne semble pas que ce compte donne une impression de fausseté ou de coulage et la gestion des Therrien paraît honnête. Le caractère le plus original des dépenses est la part importante qu'occupe l'achat des nègres; il était nécessaire pour toutes les plantations de renouveler au moins en partie ou d'accroitre la main-d'œuvre. Ces achats diminuent les bénéfices, même lorsque la récolte est normale.

31. Ces expéditions de denrées ne doivent pas être comptées dans les dépenses. 


\section{II. - LES RECETTES}

En premier lieu doivent figurer ici les: Recettes extérieures à la plantation, e'est-à-dire les recettes provenant de la maison de Saint-Marc... . 3.310 liv. 35 s. 12 d.

La vente des vins de Monsieur Seguineau, 18 barriques, venant du Bordelais................ 1.692 -

Enfin les ventes de café. Bourron étant le gérant de la caféière de Montrouy, dans les entrées de café figurent les arrivées dans le magasin général avant le départ:

85.852 livres pesant, pour. 95.706 liv. $13 \mathrm{~s}$.

Soit un total de recettes de: 100.710 liv. 8 s. $12 \mathrm{~d}$.

Il apparaît très clairement que les cafés marqués au crédit sous la dénomination entrée et sortie ne viennent pas d'une des autres caféières Seguineau, mais sont les réceptions faites par les Terrien des mains de Bourron alors gérant ou économe. Entre le gérant et le procureur, il y avait donc une comptabilité des reçus d'envoi.

Les acheteurs de café sont les frères Terrien pour leur maison de Nantes, Roux, marchand à Marseille, Cluchet, Saint-Martin et la maison Cabarrus de Bordeaux. ${ }^{32}$

Ces achats se répartissent de la façon suivante:

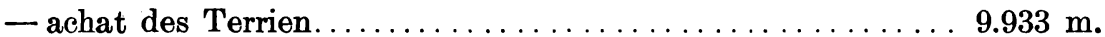

— achat du capitaine Roques. . . . . . . . . . . . . . . . 1.845 m.

- achat du Protecteur. . . . . . . . . . . . . . . . . . . 5.041 m.

de l'Auguste..................... $9.664 \mathrm{~m}$.

de l'Aimable Société...................18.960 m.

de l'Artibonite........................11.786 m.

de l'Hercule....................... 10.687 m.

— achat de Roux et Cie.........................9.073 m.

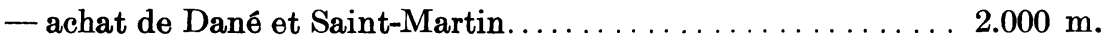

- achat de Cluchet. . . . . . . . . . . . . . . . . . . . . . . $3.812 \mathrm{~m}$.

— achat de Noël. . . . . . . . . . . . . . . . . . . . . . . . $3.051 \mathrm{~m}$.

$85.852 \mathrm{~m}$.

Les prix du café varient de 18 sous à 25 sous en 1788 ; on 1789 (d'après une lettre Terrien), il vaudra 27 sous.

La date des ventes, s'échelonne de décembre 1787 à décembre 1788. Elle ne dépendait pas de la volonté du vendeur,

32. Correspondance Navailles-Bonnas - Lettre du 14 février 1791. 
mais des occasions, des fluctuations des cours du café, du frêt des moyens de transport terrestre. Nous ignorons si l'on pouvait entreposer le café longtemps sur les caféières. Nous ne pouvons d'après les comptes d'une seule année donner une explication de la variation du prix du café. Il faudrait une suite de comptes et on n'expliquerait pas tout.

Le nombre des pieds de caféiers a pu varier au cours du XVIIIe siècle de 150.000 à 200.000 pieds.

Il est bien dit que la récolte de 1788 a été de $85.852 l$. pesant. Cela est net. Il n'y a pas chevauchement de récoltes, tout ce qui est produit en 1788 est expédie au compte des Terrien et sans doute des avances importantes consenties par eux.

\section{III. - LES REVENUS}

Pour calculer le revenu le plus précisément que l'on peut, il faut déduire du chiffre des dépenses les frais occasionnés par l'achat des noirs en 1788 (ce n'était pas là une dépense de tous les ans), soit 14.400 livres et ne pas compter comme dépense les expéditions de denrées, car cela nous masquerait les véritables bénéfices et par conséquent le revenu réel. Le chiffre total des dépenses s'établit alors à: 26.735 livres environ pour 100.710 livres de recettes, laissant un bénéfice de 73.975 livres environ. D'autre part, le capital d'exploitation pouvant être óvalué à 555.200 livres, le revenu brut serait 73.975 livres, soit à peu près de $131 / 2 \%$ à $14 \% .^{33}$

Nous connaissons le revenu brut, mais non le revenu net. Pour arriver au revenu net, il faudrait connaître certains frais de courtage, les bénéfices des négociants. Enfin, nous ne trouvons aucune perte d'animaux, aucune victime d'accidents ou d'épidermie. Évidemment 1788 est une bonne année, deux esclaves seulement sont morts en seize mois et les affaires vont encore bien. Cependant, en tenant compte de ces divers éléments, nous pourrions remmener le revenu net à $10 \%, 12 \%$ et ainsi, semble-t-il, nous serions plus près de la réalité. $12 \%$ paraît un revenu honorable et il explique la réputation des placements coloniaux.

Mais en fait, il est impossible de conclure d'après un compte annuel isolé. Il est cependant permis de s'étonner sur l'absence - fait rare - de dépenses de reconstructions ou

33. Maximilien de Navailles-Bonnas, dans ses réclamations pour obtenir l'indemnité prévue pour les anciens colons de Saint-Domingue, indique qu'au temps de sa mère, avant la Révolution, la plantation rapportait $15 \%$. 
de constructions; les absences de grosses réparations ou de remise à neuf où les procureurs et les gérants gagnaient tant. On voit bien que les frais généraux d'entretien du matériel étaient moins élevés sur une caféière, même très grande comme celle-ci, que sur une sucrerie.

Les Terrien paraissent donc honnêtes, bien qu'il y ait des dépenses que ni nous, ni le propriétaire lointain ne pouvons contrôler. Mais ils voient en gerants et en colons de Saint-Domingue. Ils veulent toujours de plus gros revenus. L'argent à Saint-Domingue doit rouler, servir à des achats.

CHAPITRE TROISIÈME

\section{L'HABITATION AU DÉBUT DE LA RÉVOLUTION}

\section{PROCUREURS ET GERANTS}

A Saint-Domingue, les propriétaires absents confiaient l'administration de leur domaine à deux représentants: le procureur et le gérant. C'est le cas de la plantation Seguineau-Navailles.

Le procureur achète les esclaves et les animaux, propose les grands travaux et les grosses dépenses, vend les produits de l'habitation, rend les comptes généraux. Il habite la ville voisine, ne vient sur la plantation que de temps en temps. Il surveille parfois plusieurs propriétés d'absents. Les procureurs étaient à l'ordinaire des colons, des commerçants qui passaient de longues années à la colonie et qui recevaient la procuration générale des propriétaires absents. Le gérant s'occupe spécialement de l'exploitation agricole, des esclaves, de la récolte. Il procède à certains achats. Il vit sur l'habitation dans la grande case. Il a des gages fixes, tandis que le procureur a généralement un pourcentage sur les recettes brutes.

Nous venons d'étudier un compte de gestion rendu par les frères Terrien à Seguineau aîné. Les Terrien remplissent correctement les fonctions de procureurs. Ils sont aussi procureurs d'autres plantations dont nous ignorons les noms, notamment Terrien jeune. Ils habitent Saint-Marc et, d'après leur correspondance, rendent de 
fréquentes visites à la caféière de Montrouy avant d'adresser, sous forme de lettres, un compte rendu de leur visite au propriétaire. Pour le détail, ils s'en remettent au gérant. Les gérants qui vivent sur la plantation sont d'abord Maillat, puis Bourron, enfin, pendant la Révolution Guchen. Nous avons sur eux très peu de renseignements.

Dans leurs lettres, les Terrien ne cessent de louer leur propre administration et ils représentent bien le type des procureurs à Saint-Domingue qui n'ont qu'un but: s'efforcer de maintenir la confiance du l'ointain maître. D'autant plus que Navailles-Bonnas, propriétaire de la plantation Seguineau, au début de la Révolution, ne se rendra jamais sur ses terres à Saint-Domingue. 1790 $)^{34}$.

(à M. de Navailles-Bonnas - Saint-Mare - 6 mai

...vos intérêts pouvant éprouver une amélioration, nous devons l'opérer; nous y sommes déterminés... Demeurez toujours persuadés que tant que nous serons chargés de vos intérêts, vous serez aussi bien représentés qu'un absent peut l'être: réalisation, goût, devoir, probité, tout vous en répond...

A chaque nouveau voyage sur la plantation, les procureurs adressent une lettre au Maitre. $1791)^{35}$.

(à M. de Navailles-Bonnas - Saint-Mare, 14 février

....Nous avons passé chez vous presque une semaine entière, nous voulons tout voir; à chaque voyage, c'est un inventaire oculaire et général qual nous faut faire, ou nous ne serions pas contents. Nous le faisons avec d'autant plus de plaisir que tout va bien...

Les rapports entre maître et procureurs sont bons. Dans cette même lettre du 14 février 1791, les Terrien expriment le désir de rencontrer prochainement Navailles-Bonnas:

...Nous en venons à vos chères lettres... Votre première débute par des choses obligeantes... dont le récit joint

34. Correspondance Navailles-Bonnas.

35. Correspondance Navailles-Bonnas. 
à votre aimable correspondance n'a pas ajouté une petite dose à notre ardente envie de faire votre connaissance. Si vous ne voulez pas Saint-Mare, acceptez au moins un rendezvous pour Bordeaux, pour août 1793...

Le gérant, qui est à ce moment Guchen, tient également de son côté Navailles-Bonnas au courant de l'état des travaux.Malheureusement dans nos papiers ne se trouve aucune lettre de Guchen. C'est un moyen de contrôle que cette correspondance du gérant avec le maître et cela doit un peu gêner les Terrien si nous en croyons la suite de la lettre du 14 février 1791:

$A$ votre prière et quoique très surabondamment, $M$. Guchen, votre gérant, a dû vous écrire pour vous dire véridiquement et librement sa façon de penser sur l'état de votre bien, son administration et sur nous-mêmes.

L'action du gérant qui demeurait sur la plantation avait une grande importance sur l'allure des travaux:

...Répondez-moi, ajoutent les procureurs, en lui parlant obligeamment de ses travaux, de son assiduite; flattez-le d'une seconde augmentation d'appointement quand vous connaîtrez une augmentation de revenus, surtout, stimulez-le à faire prodigieusement du fumier, en lui disant que quoique vous ne connaissiez pas la terre à café, vous êtes persuadé qu'aucune vieille terre au monde ne peut être recréée autrement que par le fumier et que plus le cultivateur en emploie, plus sa terre doit lui rapporter...

La nécessité des fumures est excellement mise au premier plan par les procureurs. Cette insistance est presque une nouveauté aux Antilles où l'abondance des terres remplaçait les engrais, où une terre épuisée était remplacée par des bois debout ou des savanes rases.

\section{LES ESCLAVES}

L'étude de l'esclavage colonial a suscité de tout temps de nombreuses polémiques. Nous n'interviendrons pas dans ces discussions, nous ferons simplement appel à nos textes, par malheur assez avares de renseignements sur ce point, pour connaitre la situation des esclaves sur la plantation Seguineau-Navailles. 
Le nombre des esclaves à varié sur l'habitation de 148 à 150 . C'est le chiffre de la main-d'œuvre d'une moyenne plantation. Cette question de la main-d'œuvre était essentielle autant pour l'exploitation que pour le propriétaire dont elle constituait la part principale du capital d'exploitation. La perte d'un ou de plusieurs esclaves modifiait le mouvement du travail pendant quelques mois, car on ne parvenait pas à acquérir de nouveaux travailleurs du jour au lendemain. Les procureurs estiment que 100 nègres de plus seraient nécessaires, car, disent-ils, "nous leur taillerons autant et plus d'ouvrage qu'ils en pourraient faire ${ }^{36}$."

Prix - achat - vente. - Le prix d'un esclave, en 1788, varie de 2.200 livres à 2.400 livres. Nous connaissons l'achat du 30 mars 1788: "pour le prix de 6 têtes de noirs, provenant du négrier, le marquis de Bouillé: 14.400 livres" ${ }^{37}$. Nous ignorons si, par la suite, il y eut d'autres achats.

$A g e$ - Races. - Nous n'avons également aucun renseignement, ni sur l'âge, ni sur la race de ces noirs. Nous pouvons cependant dire, d'après le tableau des naissances, qu'il y avait au moins 11 négresses dans la force de l'âge, capables de procréer. Ce chiffre était insuffisant tout de même pour renouveler l'atelier sur place sans faire appel à l'achat de noirs venus d'Afrique.

Nourriture. - Très peu de détails sur la nourriture. Les noirs, sans doute, se nourrissaient eux-mêmes directement du produit de leur jardin et ne paraissent pas avoir manqué de vivres, si nous en croyons les procureurs. $1790)^{37}$.

(a M. de Navailles-Bonnas - Saint-Mare, 6 septembre

...une apparence de vivres au-delà des besoins, laquelle apparence se changera en réalité immanquables, si nous passons quinze jours sans un vent un peu violent... $1791)^{39}$.

(à M. de Navailles-Bonnas - Saint-Marc, 14 février

...Vos magasins a vivres ont été remplis au comble. Jugez, si vos nègres pâtiront; aussi jamais un seul de dérangé de ses travaux dans le courant de l'année...

36. Correspondance Navailles-Bonnas.

37. 1788 - Compte de gestion.

38. Correspondance Navailles-Bonnas.

39. Correspondance Navailles-Bonnas. 
Gratifications. - Pour favoriser les soins donnés aux nouveauxnés, procureurs et gérants ne cessent de donner des gratifications. Ils veulent éviter la mortalité très forte durant le bas-âge chez les noirs, faute de soins, manque d'hygiène, et à cause des maladies vénériennes. Ces gratifications devaient permettre dans l'esprit des procureurs d'abaisser, dans la mesure du possible, la mortalité, car les propriétaires avaient intérêt à avoir une main-d'œuvre née sur l'habitation, ne serait-ce que pour éviter les difficultés de l'acclimatement de noirs nouvellement arrivés de l'Afrique. C'est ainsi que la négresse Hélène, pour avoir sauvé son enfant, reçoit une piastre gourde, soit 8 livres 5 ; la négresse Olive, pour la même raison, reçoit 2 piastres gourdes ou 16 livres 10 sous.

Morts - Naissances. - Le compte de gestion de 1788 nous donne un état des "naissances et mortalité" des nègres et animaux sur l'habitation de M. Seguineau aîné, du 10 août 1787 au 20 décembre 1788; savoir:

- 1787 - aout 15 - Macaya, âgé d'environ 42 ans, trouvé mort dans le grand chemin, qu'on a supposé mort d'une colique de miserere, souffrant de douleurs depuis longtemps.

- 1788 - décembre 15 - Jeanneton, âgé d'environ 30 ans, se plaignant de douleurs depuis deux ans. 2 morts $^{40}$ seulement en 16 mois.

\begin{tabular}{|c|c|c|c|}
\hline \multicolumn{4}{|c|}{ NAISSANCES } \\
\hline 1787 - Nom des Mères & $\begin{array}{l}\text { Enfants } \\
\text { nés }\end{array}$ & Morts & $\begin{array}{r}\text { Vivant au } 20 \\
\text { décembre } 1788\end{array}$ \\
\hline Marie-Louise, créole.... & 1 & 1 & “ \\
\hline Zabeth............. & 1 & 1 & “" \\
\hline Agnès........... & 1 & 1 & ." \\
\hline Marthe, créole......... & 1 & 1 & “ \\
\hline Zabeth............. & 1 & 1 & . \\
\hline Agnès. . . . . . . . . & 1 & “ & 1 âgé de 4 mois \\
\hline Marie-Louise, crø̈ole.... & 1 & 1 & " \\
\hline Olive.............. & 1 & “ & 1 âge de 2 mois \\
\hline Marthe, créole......... & 1 & 1 & " \\
\hline Hélène............. & 1 & “ & 1 âgê de 1 mois \\
\hline Minerve $\ldots . . . \ldots \ldots \ldots$ & 1 & 1 & \\
\hline Point d'animaux morts. & 11 & Morts 8 & 3 vivants \\
\hline
\end{tabular}

40. Souligné dans le texte par les frères Terrien. 
Parmi les mères qui ont conservé leur enfant, nous retrouvons les deux négresses Olive et Hélène qui ont reçu pour cela des gratifications.

Comme on peut en juger, la mortalité reste très forte durant le bas âge, puisque sur 11 naissances, il y a 8 morts. C'était un phénomène général sur l'ensemble des plantations aux Antilles.

Par la suite, les Terrien se vanteront de la faible mortalité sur la plantation, mais mortalité et natalité semblent s'équilibrer à peu près: 2 décès, 2 à 3 naissances. Ce chiffre reste insuffisant pour renouveler l'atelier, sans apport extérieur. $1791)^{41}$

(à M. de Navailles-Bonnas - Saint-Marc, 14 février

...On ne peut pas en moins perdre que nous par la mortalité; nous vous avons dit qu'il en était mort deux seulement dans le cours de 1790, dont un tué par 70 ans de vie et nous avons eu trois naissances; tous les trois sauvés et bien portants; nous n'en pouvions sauver aucun en 87 et 88 .

Les trois enfants vivants au 20 décembre 1788 n'ont donc pas vécu! Sur 11 naissances, il y a donc eu cette année là, 11 morts!

...depuis notre heureuse découverte, nous n'en perdons plus. Aussi, à notre dernier voyage, l'accoucheuse a-t-elle reçu une gratification de 4 piastres gourdes. C'est de l'argent placé à gros intérêt...

Santé. - La santé laisse parfois à désirer chez les noirs. Les années 1786-1790 ne furent point à l'Arcahaye des années de mauvaises fièvres et de varioles ${ }^{42}$; cependant, en 1790 , les Terrien notent une recrudescence de la verette.

(à M. de Navailles-Bonnas - Saint-Mare, 6 mai 1790) ${ }^{43}$.

...La malheureuse verette, après avoir quitté votre habitation, est revenue la visiter encore, trois nègres l'ont eue assez mauvaise et depuis quinze jours, cette maudite maladie a de nouveau disparu et Dieu veuille que ce soit pour longtemps! L'idée même de ce mal, dans cet atelier de 150 noirs effraye avec juste raison...

41. Correspondance Navailles-Bonnas.

42. G. Debien, "Comptes; profits, esclaves et travaux de deux sucreries de StDomingue (1774-1798)" notes d'histoire coloniale, Rev. de la Soc. d'hist. et de géogr. d'Haïti (1945), 25.

43. Correspondance Navailles-Bonnas. 
La situation s'améliore rapidement, même durant la saison des pluies en septembre. $1790)^{44}$.

(à M. de Navailles-Bonnas - Saint-Mare, 6 septembre

...Point de nègres malades dans une saison des pluies où l'hôpital a coutume d'en être plein...

En somme, l'état de santé des nègres paraît relativement bon durant ces années. D'ailleurs, M. Seguineau et les Terrien avaient passé un accord avec un chirurgien pour soigner l'atelier. C'était un usage des très grandes plantations.

Discipline. - Un bon esprit paraît avoir régné parmi les noirs de la plantation. L'absence de nombreux marrons l'atteste. Même au début de la Révolution, l'état d'esprit se maintiendra bon dans l'atelier.

(à M. de Navailles-Bonnas, 15 juillet 1790$)^{45}$.

Nous ne vous disons rien des nègres parce qu'il n'y a rien à en dire; la police et la fermeté des blancs leur ôtent jusqu'à l'idée de nous nuire. Ces mêmes motifs en imposent aux mulâtres qui ne soufflent pas le mot...

(à M. de Navailles-Bonnas, Saint-Marc, 14 février 1791$)^{46}$. ...Jamais nous ne recevons aucune plainte; à chaque voyage, les chefs viennent nous faire une petite visite affectueuse...

Il serait cependant exagéré de croire qu'il n'y eut jamais de fugitifs, de "marrons". Le marronnage n'est pas signalé dans les lettres des frères Terrien. Néanmoins, le fait que deux commandeurs sont attachés à l'exploitation et chargés spécialement de la surveillance des esclaves, laisse supposer que, de temps à autre, quelques actes d'indiscipline ont $\mathrm{pu}$ se produire. Ce serait vraiment une exception s'il n'y avait pas eu de marronnage sur la plantation.

L'absence de documents plus précis ne nous permet pas de nous prononcer sur ce point.

44. Correspondance Navailles-Bonnas.

45. Correspondance Navailles-Bonnas.

46. Correspondance Navailles-Bonnas. 
Les affranchissements. - Dans l'ensemble, les esclaves paraissent avoir été traités avec humanité: un hôpital, un chirurgien étaient là pour surveiller leur santé; une accoucheuse était également attachée à la plantation. C'était l'intérêt du maître, mais il y avait aussi des raisons d'humanité dont on ne peut diminuer l'importance.

Envers certains d'entre eux, le maître du Comté de Lognac, fait donation le 15 janvier 1789 au fils de Georges Mercure de 200 francs de rente viagère. Georges Mercure avait été le valet de chambre de Pierre Seguineau, il lui appartenait. A la date du 15 janvier 1789 , il le reconnaît "actuellement libre de sa personne."

\section{TRAVAUX, CULTURE ET RECOLTES.-}

Les lettres des procureurs nous donnent quelques renseignements sur les travaux, les cultures (nouvelles.plantations de caféiers), les récoltes au début de la Révolution. Elles permettent de reconstituer en partie l'évolution du domaine à cette époque.

Ces renseignements sont fragmentaires, incomplets. Il faut les utiliser cependant au mieux, étant donné la disparition des autres sources, notamment du journal des travaux ${ }^{47}$.

La marche des travaux paraît avoir été suivie très attentivement par les procureurs. A les entendre, jamais un nègre n'est dérangé de sa tâche.

(à M. de Navailles-Bonnas, Saint-Mare, 6 mai 1790) ${ }^{48}$.

...L'administration est telle que pas un seul nègre ne se dérange jamais de ses travaux...! "et le succès semble récompenser ces efforts: "l'administration la plus heureuse couronne tous nos travaux...

Ce sont les plantations nouvelles qui sont surtout l'objet des soins des Terrien. Sur cette vieille terre des Antilles, ils sentent la nécessité de maintenir la fertilité par l'apport de la fumure. Ils ont

47. Les journaux de travail des caféières sont très rares. On ne connaît que le Journal des travaux de la caféière Guiton de Maulévrier aux Matheux (Arch. de la Vienne En 1968) dont M. Debien prépare l'histoire.

48. Correspondance Navailles-Bonnas. 
planté 10,000 caféiers en 1788 ; ils planteront 12,000 caféiers en 1789 et 12,000 encore, en 1790 . Ces jeunes arbres sont mis:

...dans des trous de vieille terre, renouvelée par du terreau et du fumier. De là, nous pouvons juger de la progression du produit de pareilles plantations, beaucoup mieux soignées, beaucoup plus considérables faites en $89-90$ et qui le seront régulièrement chaque année...

Dans leurs lettres, les procureurs ne cessent d'insister sur la nécessité de la fumure: $1791)^{49}$

(à M. de Navailles-Bonnas - Saint-Marc, 14 février

....L'habitation est en progrès, grâce aux fumures nombreuses et répétées. Malheureusement, une grosse mouche attaque les plantations de 1788 et de $1789 .$.

Les travaux de plantation sont poursuivis inlassablement malgré les insectes qui les compromettent.

...Nos plantations ainsi faites en juin 1790 (les 12.000 derniers caféiers) nous ont charmé la vue; elles sont de la plus grande beauté.

Nous avons en train pour juin prochain une belle quantite; des trous abondants et bien faits qui se disposent à en recevoir. Nous nous proposons même de faire fumer de nouveau les arbres plantês en juin 1788 et 1789 pour soutenir leur belle venue. Ceux-ci ont été combattus cette année par un singulier ennemi. La moitié de leurs branches, de la grosseur d'un tuyau de plume ont été percées par l'aiguillon d'une grosse mouche comme une flûte. La branche n'en périssait pas pour cela; mais beaucoup viennent d'être cassées à l'endroit percé par un coup de vent du sud, un peu violent. Le mal n'est cependant pas de grande importance et n'a pas porté sur toute l'étendue des plantations...

Des préparatifs sont faits pour 1791, en vue de nouvelles plantations.

...Pour celles que nous préparons pour juin prochain (c'est-à-dire juin 1791), nous avons une pépinière de plants de caféiers d'une beau té réussie comme nous n'en avons jamais vu. Nous pouvons done d'autant mieux nous promettre de bonnes plantations...

49. Correspondance Navailles-Bonnas. 
Les récoltes confirment en partie les espérances des procureurs. Nous n'avons malheureusement que quelques chiffres. En 1788, la récolte est de 86 milliers de café (85 milliers et 852 livres); en 1789, 95 milliers; en 1790, elle n'est plus que de 65 milliers (65.000 livres pesant); enfin, en 1796, elle ne sera plus que de 41 milliers (51.000 livres pesant).

L'année 1788 peut être considérée comme une année normale, alors que 1790 et surtout 1794 sont les années exceptionnelles, étant donnée la situation dans l'île.

Dès 1790 , les procureurs paraissent déçus, malgré une récolte satisfaisante, mais ils ne perdent pas tout espoir:

(à M. de Navailles-Bonnas, Saint-Mare, 6 mai 1790) ${ }^{50}$.

...Si l'année 1790 avait été heureuse en revenus, nous pourrions dire que tout va trop heureusement bien sur notre habitation... les récoltes de 1788 et de 1789 ont trop influé sur celle de 1790 qui sera encore meilleure que nous ne l'avions craint au commencement de l'année. On a fait dans tout le courant d'avril quelques gaspillages qui la porteront à environ 65 milliers.

...Vos intérêts pouvant éprouver une amélioration, nous y sommes déterminés; la récolte de 1791 viendra et sera enfutaillée ici. Il en résultera un bénéfice, nous le démontrerons quand il le faudra, nous le prouverons non par des conjectures et des données vagues, mais par un petit exposé arithmétique...

En fait, l'arithmétique des procureurs nous paraît un peu douteuse, 65 milliers de café, en 1790 , est certes une récolte honorable, cependant nous devons tenir compte des 10.000 caféiers plantés en 1788 et qui doivent commencer à rapporter, si nous en croyons les procureurs eux-mêmes. $1790)^{51}$.

(à M. de Navailles-Bonnas - Saint-Marc, 4 septembre

...Mais ce qui prouve que nous ne jouons pas si mal, c'est que nous avons vu plus de trois milliers de café sur les jeunes arbres, plantés avec soin par nous, en juin 1788 ...

50. Correspondance Navailles-Bonnas.

51. Correspondance Navailles-Bonnas. 
10.000 pieds de caféiers avaient été plantés en juin 1788; en 1790 , le rendement est donc de 300 grammes par pied, ce qui est un rendement normal, car ces caféiers n'ont que deux ans et un pied de caféier ne rapporte placement qu'au bout de trois ans.

D'autre part, la plantation rapportait en 1788, qui est une année de bénéfices pour la plantation, 85 milliers de café. Si nous considérons que le rapport moyen d'un pied de café varie de 300 grammes à une livre, il y aurait eu sur la plantation dans les 150.000 pieds de café environ. Ces 150.000 pieds donnent, en 1788 , un rendement moyen de 650 grammes par pied environ, ce qui est un bon rendement. Si nous ajoutons à ces 150.000 caféiers, les 22.000 pieds plantés en 1788 et en 1789 , qui commencent déjà à rapporter, nous aurions en $1790,172.000$ pieds de caféiers sur la plantation ${ }^{52}$.

Avec un rendement de 300 grammes par pied, la plantation aurait da rapporter 75 milliers environ en 1790 , alors qu'elle ne rapporte que 65 milliers. Le rendement a donc baikssé en 1790, par rapport à l'année 1788 et cela malgré les plantations nouvelles. Ce rendement n'est plus en 1790 que de 300 grammes- 400 grammes par pied, rendement moyen pour l'époque aux Antilles.

Il devait y avoir certes des raisons à ce rendement moyen. 1790 n'est déjà plus une année normale et, d'autre part, il y eut "une grosse mouche qui attaque les plantations, de 1788 et de 1789". Il y a aussi des changements dans la température, des sécheresses, des pluies prolongées que nous ignorons; mais nos papiers ne nous donnent pas d'autres raisons.

Nous ignorons aussi les réactions du propriétaire en France. En 1788, d'après le compte de gestion, le bénéfice a été d'environ 75.000 livres. Mais si l'on compte d'après Hilliart d'Auberteuil ${ }^{53}$, qu'au prix de 15 sols la livre, le café doit donner 800 livres par tête de nègre, la plantation aurait dâ donner un revenu de 120.000 livres et encore le prix du café vendu a varié de 18 à 25 sols en 1788 . Si bien que sa plantation ne donnait à Navailles-Bonnas qu'un re-

52. Un bout de papier écrit de la main même de Maximilien de Navailles-Bonnas trouvé dans son livre de comptes dit: "revenus de l'année 90, 64.914 caffé et, votre habitation est composée de 150 à 200,000 cafféiers."

53. Hilliart d'Auberteuil, Considérations sur l'état présent de la colonie française de Saint-Domingue, (2 vol, Paris 1776-1777), I: 239. 
venu de 400 à 500 livres par tête de noirs et encore dans les bonnes années.

Nous nous tenons toujours dans une moyenne honorable, mais sans plus. Navailles-Bonnas fit-il part de son mécontentement aux procureurs? Nous l'ignorons. Mais si, récriminations du maitre il y eut, ces récriminations ne paraissent pas avoir troublé les frères Terrien. Ces derniers espèrent toujours de meilleures récoltes pour les années suivantes et ils se fondent sur le fait que les pieds de caféiers récemment plantés vont commencer à rapporter pleinement. $1791)^{54}$

(à M. Navailles-Bonnas - Saint-Mare, le 14 février

...oui, Monsieur, nos caféiers, plantés avec précaution, dans la vieille terre, en juin 1788, sans autant de fumier, sans autant de soin que nous avons pu donner en juin $\mathbf{1 7 8 9}$, rapportent déjà joliment. Le gérant en a déjà récolté environ 2.500 livres et nous en avons bien vu autant encore sur les mêmes arbres; voilà done 5 milliers de café réels, c'est le produit de 10.000 caféiers plantés en juin 1788 (sur ces jeunes caféiers, le rapport est done de une demi-livre par pied); environ 12.000 l'ont été et beaucoup mieux en juin 1789; autant en juin 1790 et toujours ce sera de même, sans interruption, plutôt plus que moins, parce que nous faisons travailler de manière à avoir plus de fumier...

Le rendement ici plus élevé parce que les pieds sont jeunes, atteint au bout de trois ans le rendement normal et aussi grâce à l'utilisation de la fumure ${ }^{55}$.

“...jugez par les progressions successives de ces produits, si on peut craindre de voir tomber les revenus; nous répondons du contraire et nous nous flattons même de les augmenter..."

L'espoir des procureurs pour un meilleur rendement repose donc sur les pieds de jeunes caféiers qui rapportent une moyenne d'une demi-livre par pied. Mais les plantations de 89 et de 90 n'ont pas encore donné. La récolte de 1790 sera de 65 milliers de café, soit un rendement moyen de 425 grammes par pied. Et cela malgré: "la pluie extraordinairement prolongée qui a empêché le

54. Correspondance Navailles-Bonnas.

55. Le café commençait à entrer en rapport au bout de trois ans, donnait son plein vers la huitième année et durait èn moyenne 20 ans. 
café de sécher promptement... et empêche la denrée d'être aussi belle que nous nous l'étions promis."

En somme, beaucoup d'efforts paraissent avoir été tentés par les procureurs pour redonner à la caféière un revenu plus important en augmentant le rendement. Des plantations de caféiers: 34.000 pieds de 1788 à 1790, laissent supposer que les Terrien voulaient entièrement renouveler la plantation en sujets jeunes susceptibles de donner un rendement maximum.

Le rendement reste pendant ces années juste moyen et varie de 300 à 450 grammes par pied, ce qui est assez peu si l'on songe qu'à Jérémie, par exemple, à la même époque, une caféière de deux ans donnait 2 livres $1 / 2$ de café par pied et par an et que l'habitation Faure à Torbeck donnait 2 livres $1 / 4$ par pied ${ }^{56}$.

56. Moreau de Saint-Méry, op. cit. II: 812 et II: 276. Le rendement du café était très variable à Saint-Domingue. Quelques exemples nous donneront une idée de ces variations. C'est la raison pour laquelle une extrême prudence s'impose dans les conclusions. Hilliart d'Auberteuil, op. cit. I: 239 dit: "Le café dans un sol profond pourrait au prix de 15 sols la livre, donner environ 800 livres par tête de nègres, tous frais déduits, hors ceux du transport qui sont considérables et qu'on ne peut évaluer parce qu'ils augmentent ou diminuent à proportion de l'éloignement."

On a trouvé des caféiers donnant 2 livres 1/4 par pied (Moreau de Saint-Méry cite le cas de l'habitation Faure, a Torbeck (II: 276) 30.000 pieds $=75$ milliers) - à Jérémie une caféière de 2 ans donnerait 2 livres $1 / 2$ de café par an. (Moreau de Saint-Méry, II: 812).

- Un petit planteur des quartiers des Grands Bois au Mirebalais (arch. Vienne E. 1285) écrit le 15 avril 1770 que 150.000 pieds donnent environ 100.000 livres pesant par an, parfois 120 milliers; le 14 mars 1773 il dit que 87.000 pieds donnent 80 milliers.

- La caféière Volant qui espérait de 120 à 130 milliers en 1788 en aura moins de 50 milliers (bibl. La Rochelle, Ms. 855. Papiers Bontin, 30 sept 1788) - A titre de comparaison, dans l'état de Sao-Paulo, au Brésil, en 1909-1910, 1.000 pieds $=$ 63,3 arrobas $(1$ arroba $=15$ kilogs $)=949 \mathrm{k}$. 5 , soit un arbre donne $0 \mathrm{k} .949=2$ livres.

Wimpffen, Voyage à Saint-Domingue pendant les années 1788-1790 (2 vol., Paris, an V), 1: 57, note 1 dit qu'un carreau de caféier donne 1 millier de café, soit environ 100 écus. Mais un carreau pouvait porter de 3. à 5.000 pieds; d'où une très grande incertitude (Girod-Chantrans, Voyage d'un Suisse dans differentes colonies d'Amérique - Neufchâtel 1796, in-16, p. 380). - à Port-de-Paix, au canton de Moka, en 1784, sur l'habitation Bonseigneur, 25 nègres récoltèrent 90 milliers (Moreau de Saint-Méry, 1:714). - En Guyane hollandaise, 2 noirs par 3 acres, à 450 pieds par acre, 1 pied donnant $11.1 / 2=740 \mathrm{gr}$. (G. Pinckard, Notes on the West Indies.) London 1806, 3 vol. in 8o, III, 403) - Toujours Girod-Chantrans (p. 285) dit qu'un caféier donne une $1 / 2$ livre par an et p. 286: "Si l'on suppose un prix moyen de 8 sols la livre; frais de transport payés, on trouverait qu'une caféterie peut rendre de net le $8 \%$, pourvu qu'il n'arrive aucune maladie extraordinaire ni sur les nègres ni sur le bétail."

- Notons que tous ces chiffres ne sont pas des chiffres de comptabilité. Les nôtres paraissent plus sûrs. 


\section{PROJETS D'ACHAT. -}

Pour augmenter les revenus, une solution pourrait être envisagée: l'achat d'une autre plantation. C'est pourquoi en 1791, les procureurs présentent à leur maître un projet d'achat. $1791)^{57}$.

(à M. de Navailles-Bonnas. - Saint-Mare, 14 février

...Nous vous remettons ci-joint deux lettres de particuliers dont nous visons la terre à votre intention... Nous avons employé toute la soirée à visiter sa terre. C'est la seule à votre convenance... Elle a mauvaise mine, parce que tout y est négligé, mais le fonds est bon, à force de bras, on rétablira avec succès. Il faut une heure à vos nègres pour y aller et en revenir de chez vous.

Jugez du grand avantage de pouvoir donner souvent et facilement de grands coups de mains sur une petite étendue de terre.

Voicy nos idées à peu près sur cette acquisition et la manière d'en tirer parti; cela peut valoir environ 260 mille livres, peut-être mieux avec 25 nègres. Nous en complèterions 40 par 15 bons, choisis dans votre atelier; nous remplacerions ces 15 par un achat commode fait en deux années et à terme d'un an pour le paiement de moitié.

Cette habitation fait environ 20 milliers de café qui payeraient à plus de $10 \%$ les intérêts des premiers termes payés, déboursés courants, dépenses d'administration, etc...

Nous aurions certainement de longs termes pour les $3 / 5$ du montant de l'achat et lorsque l'époque de l'échéance de ces termes arriverait, les produits bien augmentés par deux et trois années de travaux aideraient à en payer une bonne partie.

C'est une bonne affaire... en raison du voisinage de l'objet acquis...

Cette caféière dont le projet d'achat est soumis à NavaillesBonnas n'était pas très éloignée; elle produisait environ 20 milliers de café. Il y avait 25 nègres et 35 à 40.000 pieds de café environ. Le rapport $10 \%$ parait normal, le prix de vente légèrement gonflé. Cette acquisition aurait permis une augmentation du produit en café et donc un accroissement du revenu.

57. Correspondance Navailles-Bonnas. 
D'après nos papiers, aucune suite ne parait avoir été donnée à ce projet, pas plus d'ailleurs qu'en 1788 au projet d'achat d'une sucrerie, la sucrerie de Lanzac au Montrouy. Et pourtant ce dernier projet eut été la réalisation de l'éternelle ambition des colons: devenir sucriers!

\author{
(d suivre) \\ Roger M Assio, professeur \\ Collège Vic-Bigorre, \\ Hautes-Pyrénées, France.
}

- AVIS - La Revue d'Histoire de l'Amérique française vit de ses abonnements et rien que de ses abonnements. Elle compte que vous renouvellerez le vôtre, dès ces jours-ci, pour ne pas manquer le numéro de juin 1952, ler de la 6ième année. 